\title{
Transient heat transfer analysis for brake disks using SPH
}

\author{
Guiming Rong ${ }^{1 . a}$ and Hiroyuki Kisu ${ }^{2 . b}$ \\ ${ }^{1}$ Graduate School of Science and Technology, Nagasaki University, 1-14 Bunkyo-machi, \\ Nagasaki 852-8521, Japan \\ 2 Department of Mechanical Systems Engineering, Nagasaki University \\ ${ }^{a}$ rong@nagasaki-u.ac.jp, ${ }^{b}$ kisu@nagasaki-u.ac.jp
}

Keywords: Smoothed particle hydrodynamics, Transient heat transfer, Ventilated disk brake

\begin{abstract}
Smoothed particle hydrodynamics (SPH) is a mesh-free numerical approximation technique for simulating various physical problems. A calculation system for transient heat transfer problem by SPH has been improved to deal with various boundary conditions and several model calculations are performed to verify it. As a practical application, the transient temperature field of a brake disk under emergency braking is analyzed. Both solid and ventilated disks are modeled with a moving heat source on the sliding surface. The numerical results show that the temperature sharply fluctuates because of the cyclic loading. Improvement of the calculation model is also discussed.
\end{abstract}

\section{Introduction}

SPH can easily handle large deformation problems or complex problems, such as those involving both solids and liquids [1]. The authors are establishing a numerical analysis system for coupled solid-fluid problems using SPH. In this study, we analyze the transient heat transfer of brake disks.

Now the disk-type brake is widely used in automobiles. The thermal stress repeatedly occured during its operation significantly affect the working life of the break. Therefore, many studies have conducted thermal-stress analyses, typically by the finite element method (FEM) using commercial software, and only the solid type has been analyzed (e.g., [2]). In this paper, we analyze both the solid and ventilated types of disk brakes, examine the temperature distributions during operation, and clarify the effect of fins in the ventilated type disk.

\section{Formulation of SPH for Transient Heat Transfer Problem}

The formulation of SPH has been stated in many papers and books [1,3]. Due to space limitations, only those directly concerned with transient heat transfer are given here. For an explicit solution using $\mathrm{SPH}$, the governing differential equation for the transient heat transfer problem is as follows [3]:

$$
\frac{T_{i}^{n+1}-T_{i}^{n}}{\Delta t}=2 \frac{\kappa_{i}}{\rho_{i} c_{i}} \sum_{j=1}^{N} \frac{m_{j}}{\rho_{j}} \frac{T_{i}^{n}-T_{j}^{n}}{x_{i j}} \nabla_{j} W_{i j}\left(x_{i j}, h\right),
$$

when the conductivity $\kappa$ is a constant, and there is no heat source in the domain. In this equation, $T$ is the temperature, $t$ is the time, $c$ is the specific heat capacity and $\rho_{j}$ is the density. Furthermore, $m_{j}$ is the mass of particle $j, N$ is the number of particles, $W\left(x_{i j}, h\right)$ is a smoothing kernel function which is continuous in its influence domain with $h$ as the smoothing length, and $x_{i j}$ as the magnitude of the distance between particles $i$ and $j$, and $\nabla_{j} W_{i j}=\frac{x_{i}-x_{j}}{x_{i j}} \frac{\partial W_{i j}}{\partial x_{i j}}$. In this study, a cubic function is selected as the kernel function [1].

Two types of boundary conditions, the Neumann $\left(\kappa \partial T_{b} / \partial n=q_{0}\right)$ and Robin $\left(\kappa \partial T_{b} / \partial n=\Psi\left(T_{s}-T\right)\right.$, are used in our calculation, where $q_{0}$ is the heat flux and $\Psi$ is the convective heat transfer coefficient. An isothermal boundary is simply letting the temperature of particles on the boundary keep a specified value [3]; therefore, this boundary is not tested here. For the Neumann boundary condition, the SPH formulation is expressed by

$$
\frac{\partial T_{i}(\boldsymbol{x}, t)}{\partial n}=\zeta \sum_{k=1}^{D} \sum_{j=1}^{N} \frac{m_{j}}{\rho_{i}}\left(T_{i}-T_{j}\right) \frac{\partial W_{i j}}{\partial x_{i}} \boldsymbol{n}^{k}=\frac{q_{0}}{\kappa},
$$


where $\boldsymbol{x}$ is a point on the boundary and $\boldsymbol{n}^{k}$ is the direction cosine in the $k$ direction; $\zeta$ is a coefficient for the supporting domain on a boundary that is not a circle.

For the Robin boundary condition,

$$
\frac{\partial T_{i}(\boldsymbol{x}, t)}{\partial n}=\zeta \sum_{k=1}^{D} \sum_{j=1}^{N} \frac{m_{j}}{\rho_{i}}\left(T_{j}-T_{i}\right) \frac{\partial W_{i j}}{\partial x_{i}} \boldsymbol{n}^{k}=\frac{\Psi}{\kappa}\left(T_{s}-T_{i}(\boldsymbol{x}, t)\right)
$$

is adopted, where $T_{S}$ is the temperature of the surroundings.

\section{Model Calculation for Transient Heat Transfer Problem}

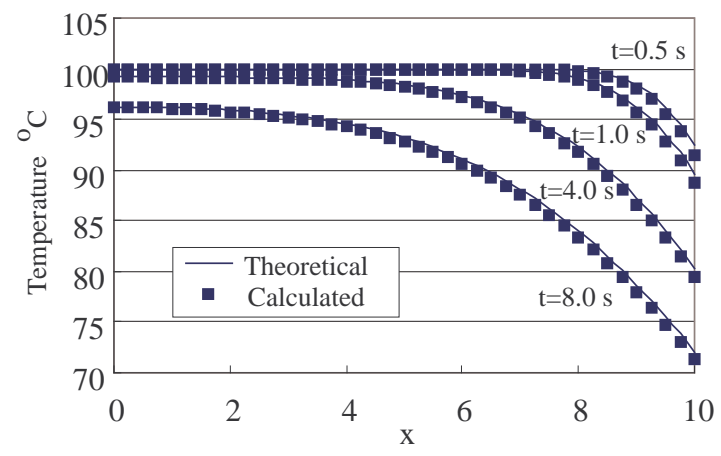

Figure 1: Distribution of $T$ according to time for the line at $y=5 \mathrm{~cm}, z=5 \mathrm{~cm}$

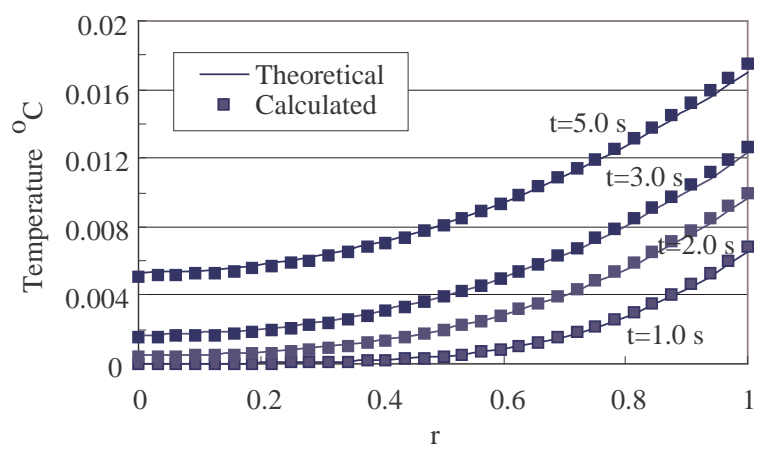

Figure 2: Distribution of $T$. Flux $q_{0}=10$ is given for the boundary of the circle

Because we are analyzing a three-dimensional problem, and primarily use the boundary conditions of Eqs. 2 and 3, two model calculations are performed and their results are compared with the theoretical results. The first calculation is mainly for testing Eq. 3. The domain is a cubic shape of $10 \times 10 \times 5 \mathrm{~cm}$; particle number of $N=4851$ is used for the domain, which is evenly divided by $20 \times 20 \times 10$; so that one particle area is approximately $m_{i} / \rho_{i} \approx 0.125$ and $h=0.5 \mathrm{~cm}$. Time step $\Delta t=0.005 \mathrm{~s}$ and $\alpha=\kappa_{i} /\left(\rho_{i} c_{i}\right)=1 \mathrm{~cm}^{2} / \mathrm{s}$ are adopted. Equation 3 is applied to $x=10, y=10$ for $\Psi=0.1 \mathrm{~W} / \mathrm{cm}^{2} \mathrm{~K}$; the other boundaries are adiabatic. The initial condition is $T(x, y, z, 0)=T_{0}=100^{\circ} \mathrm{C}$.

Figure 1 illustrates the results at times $t=0.5,1,4,8 \mathrm{~s}$ of the analytical solution, compared with the $\mathrm{SPH}$ results at the line $y=5, z=5 \mathrm{~cm}$. The largest error of the calculated results is approximately $3 \%$ in comparison with the theoretical results.

The next calculation uses the Neumann condition. We let flux $q=10 \mathrm{~W} / \mathrm{m}^{2}$ apply to the cylindrical surface of a cylinder with radius $1 \mathrm{~m}$ and height $z=0.25 \mathrm{~m}$, where $T_{0}=0^{\circ} \mathrm{C}$. Also adopted are $\rho=7860$ $\mathrm{kg} / \mathrm{m}^{3}, \kappa=41.26 \mathrm{~W} / \mathrm{m} \mathrm{K}, c=0.1146 \mathrm{~J} / \mathrm{kg} \mathrm{K}$. The particle number is $N=25821$. The results are also compared with the SPH results in the radial direction for $z=0.125 \mathrm{~m}$. The largest error is almost the same as in the previous example. The influence of the dividing number and time step on the precision are not discussed here due to the space limitations. Similar calculations of 2D problems are also performed and no significant differences are found when compared to the results of the 3D problems. In addition, in an application to an inhomogeneous domain, as well as to the problem of coefficients changing with the temperature, our calculations are also successful. From these calculations, we can say that the proposed system is reliable for thermal analysis.

\section{Analysis Model for Brake Disks}

The main parts of a disk brake system are the disk (or rotor) and two friction pads set next to the two sides of the disk. When the brakes are applied, the pads put pressure on the two working faces of the rotating disk; then, the friction force increases and slows the rotation. In this process, most of the mechanical energy is transformed to thermal energy. Since we are investigating the difference 
Table 1: Dimension and material properties of the brake disks

\begin{tabular}{cccccc}
\hline Inner radius & Outer radius & Thickness & Density & Specific heat & Thermal conductivity \\
\hline $85[\mathrm{~mm}]$ & $128[\mathrm{~mm}]$ & $16[\mathrm{~mm}]$ & $7031\left[\mathrm{~kg} / \mathrm{m}^{3}\right]$ & $495[\mathrm{Nm} / \mathrm{kgK}]$ & $56.72[\mathrm{~W} / \mathrm{mK}]$ \\
\hline
\end{tabular}

Table 2: Analysis conditions by SPH for the disks

\begin{tabular}{cccccc}
\hline Frictional coef. & Pressure & Initial speed & Initial temp. & Particle no. of solid type & Time step \\
\hline$\mu=0.38$ & $2.38[\mathrm{MPa}]$ & $\omega_{0}=96.2 /[\mathrm{s}]$ & $40\left[{ }^{\circ} \mathrm{C}\right]$ & 36225 & $0.0005[\mathrm{~s}]$ \\
\hline
\end{tabular}

in the temperature distribution of the solid and ventilated types of disks, let us make the following simplifications: 1. All the mechanical energy is transformed to thermal energy. Since the energy is absorbed by both the disk and pads, we suppose $86 \%$ of the energy is absorbed into the disk; 2 . In the braking process, the rotating speed of the disk is calculated by $\omega=\omega_{0}-a t$ ( $a=20.38$, a constant); 3. The solid and ventilated disks have the same dimension in the radius direction but different in thickness (Table 1 shows the thickness of the solid one, it is 22 for ventilated type); however, they have the same physical properties, and are analyzed with the same parameters, shown in Tables 1 and 2 (except for the particle number). The pad, which has an inner radius of $81.5 \mathrm{~mm}$ and outer radius of $125 \mathrm{~mm}$, covers a range of 60 degrees, as shown in Fig. 3, and the pressure is the same everywhere at every time step, except for the surrounding edges.

The solid and ventilated disks have a similar shape, which can be considered to be symmetric about the central crossing plane of the disk, and so we only need to analyze the half domain of the disk and let the central crossing plane be adiabatic. Figure 3 is an analysis model of the solid disk. The boundary conditions are set so that the central crossing plane is an adiabatic boundary and the outer cylindrical surface is convective. For the working surface, the range which contacts the pad satisfies Eq. 2, where flux $q$ is calculated by

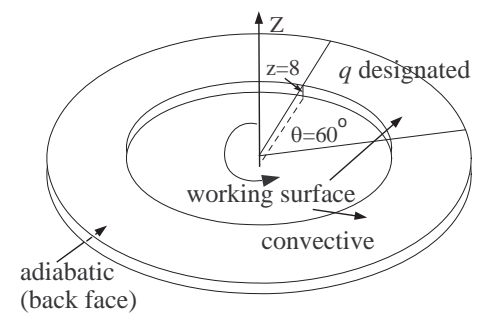

Figure 3: Model for a solid disk

$$
q\left(r_{i}, t\right)=\mu p(x, y, t) v(x, y, t)=\mu p(x, y, t) r_{i} \omega(t) .
$$

This range is always changed during the braking process. The working surface not in contact with the pad satisfies Eq. 3, where $\Psi$ is determined by the experimental formulas of Rudolf Limpert [4].

A ventilated type disk usually can be considered as two thin solid disks nipped with a set of ventilated fins. (To see the effect of the fins, neither a slit nor hole is used.) Therefore, the ventilated type disk can also be considered as having a symmetric shape. For comparison, we let one side of the outer disk have half of the thickness of the solid model. For simplicity, we set 12 fins in our model, as shown in Fig. 4, each of which is 45 degrees in the radial direction. Therefore, 2700 additional particles are needed for the fins. It should be noted that this arrangement has little influence on the calculations because this is not a solid-fluid problem.

First, the solid type disk is analyzed. The temperature distribution at the end of braking is shown in Fig. 5, and its variation during the entire braking process is shown in Fig. 7, for several points of different radii. Here, we show the profiles at $z=6 \mathrm{~mm}, 2 \mathrm{~mm}$ from the working surface. We can see that at this cross section the profiles are still increasing zigzag curves, just as those at the working surface [2], because every point on this surface where the pad passes undergoes a heating-cooling cycle. However, the range of fluctuation is smaller than at the working surface and almost disappears at the cross section of $z=4 \mathrm{~mm}$, where the temperature increases smoothly.

The temperature distribution of the ventilated type is shown in Fig. 6. Here, the so-called "hot band" (a narrow ring of high temperature) [2] has disappeared; instead, "hot islands" appear, and the number of these corresponds to the number of fins. The relation of the profiles to time is similar to the 


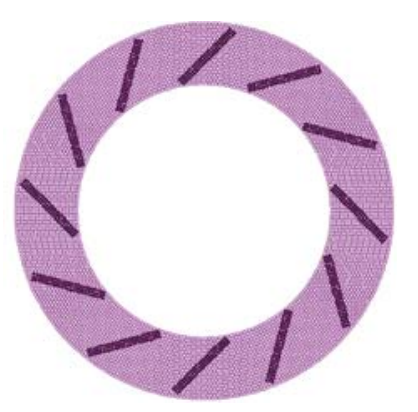

Figure 4: Arrangement of fins for a ventilated disk

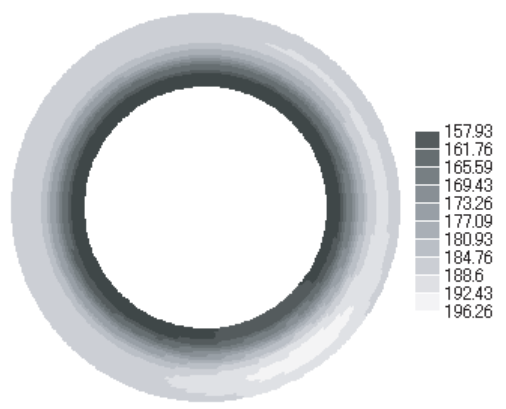

Figure 5: Temperature distribution of the solid type

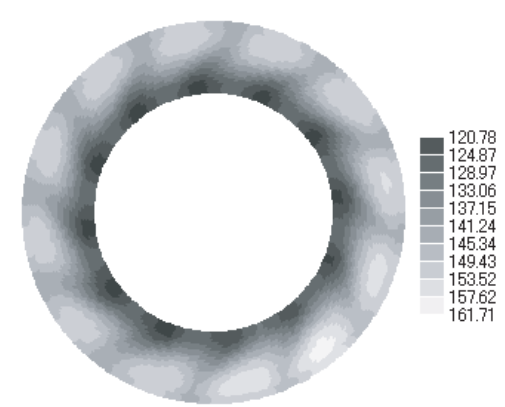

Figure 6: Temperature distribution of the ventilated type

profiles of the solid disk except at the end of braking, at which time the temperature is approximately 40 degrees, or 25\% lower than that of the solid disk (see Fig. 8). Furthermore, comparing the tendency of the two profiles, we notice two characteristics: 1 . Up to the time $t=1.0 \mathrm{~s}$, the temperature increases in the two types are almost the same. After that, the ventilated type increases more slowly; 2. In the ventilated type, the temperature generally remains within a definite range of fluctuation from time $t=3.0 \mathrm{~s}$ to the end of braking, while the temperature continues to increase slowly in the solid type.

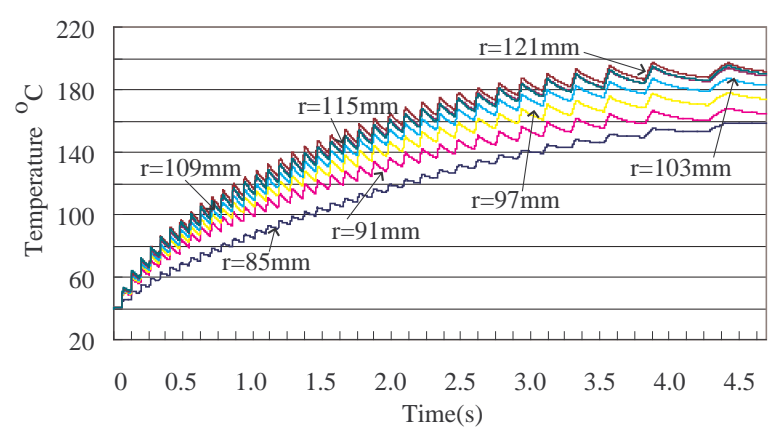

Figure 7: Relation of $T$ with time at various radii in the solid type

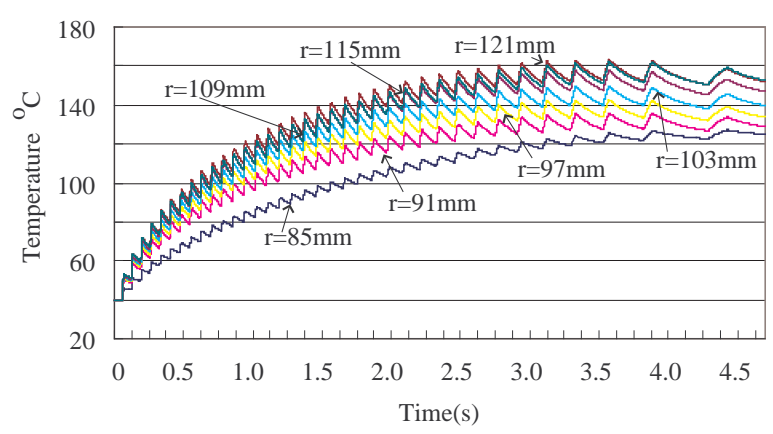

Figure 8: Relation of $T$ with time at various radii in the ventilated type

\section{Conclusions}

Two model calculations are performed in this study and the validity of the proposed numerical system is demonstrated. Next, both solid and ventilated type disks are analyzed and their temperature distributions are compared. We will continue studying ways to extend the system's ability to analyze thermal-stress and coupled fluid-solid problems, in order to improve its ability to generate optimized designs.

\section{References}

1. G. R. Liu and M. B. Liu, Smoothed Particle Hydrodynamics-A Meshfree Particle Method, World Scientific Publishing (2003).

2. C. H. Gao, J. M. Huang, X. Z. Liu and X. S. Tang, Trans ASME, Vol. 129, p. 536 (2007).

3. P. W. Cleary and J. J. Monaghan, Conduction Modelling using Smoothed Particle Hydrodynamics, J. Compu. Phys. 148, pp. 227-264 (1999).

4. R. Limpert, Brake Design and Safety, Science of Automotive Engineers Inc., Warrendale, PA, (1992). 